\section{Particularidades del uso de las Tecnologías de la Información y la Comunicación en la Educación}

Particularities of the Use of Information and Communication Technologies in Education

\section{RESUMEN}

Las Tecnologías de la Información y la Comunicación (TIC) han experimentado un crecimiento abrumador a partir de la declaratoria de pandemia y el cierre forzado de las instituciones de educación en el mundo. El presente artículo se interesa en la problemática de las TIC respecto a la educación en América Latina, centrándose en la desigualdad de género, el nivel de capacitación de los docentes, así como el acceso a internet; para este propósito se empleó un enfoque metodológico de carácter cualitativo no experimental dado que se realizó un análisis empírico (inductivo). Se encontró que la brecha digital de género todavía es un factor recurrente, que limita y condiciona no solo la educación sino el futuro laboral de las mujeres, también se pudo destacar que la capacitación de los docentes todavía necesita abordarse desde otras perspectivas dado el carácter exógeno de las TIC en la educación. Finalmente se evidencia la dificultad del acceso a internet en la región, y que invita a pensar en estrategias que permitan superar estas desigualdades y problemáticas.

Palabras claves: Educación; TIC; América Latina; desigualdad; brecha digital

\section{ABSTRACT}

Information and Communication Technologies (ICT) have experienced impressive growth since the declaration of the pandemic and the forced closure of educational institutions in the world. This article is interested in the problem of ICTs in education in Latin America, focusing on gender inequality, the level of teacher training and Internet access; for this purpose, a non-experimental qualitative methodological approach was used, given that an empirical (inductive) analysis was carried out, it was found that the gender digital gap is still a recurrent factor, which limits and conditions not only education but also the labor future of women, and it was also noted that teacher training still needs to be approached from other perspectives given the exogenous nature of ICT in education. Finally, the difficulty of access to the Internet in the region is evident, which invites us to think of strategies to overcome these inequalities and problems.

Keywords: Education; ICT; Latin America; inequality; digital divide
RELIGACIÓN

REVISTA DE CIENCIAS SOCIALES Y HUMANIDADES JOURNAL OF SOCIAL SCIENCES AND HUMANITIES REVISTA DE CENCIAS SOCIAIS E HUMANAS

INFORMACIÓN:

http://doi.org/10.46652/rgn.v7i31.898 ISSN 2477-9083

Vol. 7 No. 31, 2022. e210898

Quito, Ecuador

Enviado: noviembre 08, 2021

Aceptado: enero 10, 2022

Publicado: enero 22, 2022

Publicación Continua

Sección General | Peer Reviewed

\section{AUTORAS:}

(D) Bélgica Cecilia Arias Macías

Universidad de Especialidades Espíritu Santo - Ecuador

barias@uees.edu.ec

(D) Carmen Luisa Soto Montoya

Universidad de Especialidades Espíritu Santo - Ecuador

carmensoto@uees.edu.ec

(1) Emma Elizabeth Sacón Martinez Universidad de Especialidades Espíritu Santo - Ecuador

emmasacon@uees.edu.ec

Conflicto de intereses

Las autoras declaran que no existe conflicto de interés posible.

Financiamiento

No existió asistencia financiera de partes externas al presente artículo.

Agradecimiento

$\mathrm{N} / \mathrm{A}$

Nota

El artículo no se desprende de un trabajo anterior.

ENTIDAD EDITORA 


\section{Introducción}

El surgimiento del internet y el avance de las nuevas tecnologías empleadas en los distintos campos de la sociedad permearon rápidamente el campo de la educación. Desde la década de 1990, un número importante de iniciativas e investigaciones educativas se han orientado hacia la integración de las Tecnologías de la Información y de la Comunicación (TIC) en los distintos niveles escolares. Se han probado varios enfoques, entre ellos destacan, en primer lugar, el desarrollo de infraestructuras tecnológicas en las escuelas y, en segundo lugar, la producción e infusión en las escuelas de sofisticadas herramientas basadas en las TIC para la enseñanza y el aprendizaje (Jimoyiannis \& Komis, 2007). Los motivos y argumentos a favor de la implementación de las TIC provienen de muchas direcciones; tanto de los defensores dentro de las escuelas como, más a menudo, desde el exterior por parte de los desarrolladores de software y hardware, y del gobierno (Cuban, 1986, 2002; Selwyn \& Fitz, 2001). En los últimos 30 años ha habido un impulso continuo con la intención de integrar los ordenadores y las TIC en las escuelas.

Con la masificación del Internet, se está configurando una nueva sociedad que no sólo consume conocimiento, sino que también lo crea (Jiménez-Becerra \& Segovia-Cifuentes, 2020). Investigaciones han demostrado que el uso de las TIC en la educación puede aumentar la motivación de los alumnos y profundizar en su comprensión, promover el aprendizaje activo, colaborativo y permanente, ofrecer recursos de trabajo compartidos y un mejor acceso a la información, y ayudarles a pensar y comunicarse de forma creativa (Jonassen, 2000; Webb, 2005). En otras palabras, las TIC parecen cambiar la naturaleza misma de la enseñanza y el aprendizaje. Con las nuevas tecnologías emergentes, la profesión docente podría evolucionar desde un énfasis en la instrucción centrada en el profesor hasta entornos de aprendizaje interactivos centrados en el alumno.

En la actualidad, el impacto real de la integración de las TIC en las prácticas cotidianas del aula constituye una cuestión esencial. En la práctica, no obstante, los planes de estudio y los enfoques pedagógicos establecidos siguen siendo básicamente los mismos, mientras que la tecnología suele estar infrautilizada o mal integrada en el aula (Cuban, 2001; Ofsted, 2004). Esto plantea inquietudes respecto a los resultados de las iniciativas, si éstas son pertinentes, si son evidentes los logros de los alumnos en cuanto a la capacidad de utilizar las TIC, así como en la aplicación de sus habilidades y conocimientos a otras materias del plan de estudios.

Aunque el acceso a las TIC en los hogares ha crecido rápidamente, tanto para los alumnos como para los profesores, y la infraestructura de las TIC en los centros educativos ha mejorado sustancialmente en los últimos años, los profesores no parecen hacer un uso eficaz de las herramientas TIC en su enseñanza (Russell et al., 2003; Waite, 2004). Parece que sus actitudes y su nivel de conocimientos siguen siendo un obstáculo para que adopten y utilicen eficazmente las TIC (Dexter et al., 1999; Lang, 2000; Pelgrum, 2001).

Las TIC pueden contribuir a difuminar las fronteras entre los distintos contextos de aprendizaje en que participan los alumnos (Engel y Coll, 2022). Es bajo esta premisa que el presente artículo busca explorar las posibles problemáticas en el uso de las TIC posterior a la declaratoria del SARS-CoV-2 como pandemia y cómo ésta afectó al campo educativo. 


\section{Metodología}

El presente artículo empleó un enfoque metodológico de carácter cualitativo no experimental dado que se realizó un análisis empírico (inductivo), el corpus objeto de estudio se recolectó en un solo momento y tiempo determinados (2020-2021). Además, el propósito de la presente investigación es describir, empleando la interpretación y análisis de los artículos que conforman el corpus.

El corpus fue seleccionado a partir de artículos que aborden la relación TIC y educación durante los años 2020 y 2021, haciendo énfasis en los estragos por la pandemia en curso. Se seleccionar cuatro campos para el análisis; género, acceso a las TIC, capacitación en cuanto al uso de TIC, así como la funcionalidad/pertinencia de las mismas.

Debido a su componente inductivo, la investigación presenta un carácter exploratorio, por lo que mayores conclusiones requerirá necesariamente una ampliación de componentes y un abundante corpus.

\section{Desarrollo}

El impulso que ha recibido la implantación de las TIC en el ámbito educativo, ha implicado una inversión cada vez más grande desde sectores privados y estatales, así como el desarrollo de nuevas herramientas que constantemente refuercen las prácticas educativas y de esta manera mejorar los niveles educativos. Sin embargo, los objetivos no se han cumplido a cabalidad, principalmente debido a la falta de tecnología y software en las escuelas y la limitada experiencia de los profesores en el uso de las TIC, hasta otros factores como las creencias y los conocimientos de los profesores sobre cómo integrar las TIC en la enseñanza.

La brecha entre la retórica optimista que rodea el uso de las TIC en la educación y el nivel actual de integración de las TIC en los entornos educativos ha inspirado a los investigadores a centrarse en los profesores y estudiantes en las dificultades que encuentran para integrar las herramientas TIC en sus prácticas en el aula. A inicios del año 2020 y con la declaratoria de pandemia, el cambio de modalidad de estudios obligó a la mayoría de instituciones de enseñanza a modificar sus planes y propuestas con TIC; en este proceso se evidenciaron diversas limitantes y beneficios, que sin embargo deben analizarse detenidamente para identificar posibles vías de perfeccionamiento.

\subsection{Brecha digital de género}

Así como el desarrollo económico a nivel mundial ha generado desigualdades, también en el campo tecnológico se han podido experimentar cambios que han dejado de lado a ciertos grupos, personas y ámbitos de la sociedad. Para nuestro propósito, la brecha digital de género, ha sido analizada desde hace poco tiempo atrás. Según Prendes-Espinosa et al. (2020), es a partir de 2017 cuando se experimenta un aumento en la generación de investigación sobre esta problemática de igualdad de género a través de las TIC. Ese es un factor adicional que demuestra la creciente preocupación por la desigualdad de género en este ámbito, puesto que como advierte el informe del World Economic Forum (WEF, 2016), "El futuro de los empleos", será el avance de la tecnología, de la inteligencia artificial, así como el conocimiento y acceso 
a ella, el factor determinante que deje a 7.1 millones de personas sin trabajo debido a factores como la automatización o desintermediación. Estas plazas de trabajo son mayoritariamente cubiertas por mujeres, por lo que serán éstas quienes caigan en el desempleo, ampliando aún más la brecha digital de género.

Por otra parte, a pesar de los esfuerzos por incrementar el acceso y uso de la tecnología por parte de las mujeres, todavía es evidente la desigualdad en este campo. Para Pavez (2015), la brecha de género comienza con la falta de inclusión que tienen las niñas al momento de usar TIC. En Arredondo et al., (2019) se recogen datos de Schaaper (2013) que demuestran que "existen 1.3 billones de mujeres usuarias de internet, en comparación con 1.5 billones de hombres. Esta diferencia puede ser de dos por ciento en países desarrollados y de 16 por ciento en países en vías de desarrollo" (p. 143).

Otro factor que alimenta la desigualdad es la violencia a través de las TIC, que aleja a la mujer del acceso y uso de estos instrumentos. Esta violencia es ejercida por ambos géneros, y acarrea riesgos a nivel psicológico, físico y social del individuo, sin embargo, es la mujer quien sufre mayormente este tipo de violencia a diferencia del género masculino (Domínguez \& Portela, 2020).

Con el surgimiento de la pandemia por Covid-19, la relación entre educación y desigual de género mediada por TIC ha experimentado cambios que todavía son difíciles de analizar debido al tiempo y pocos estudios realizados. De ahí que sea parte del objetivo de este artículo el posicionar las problemáticas que deben trabajarse desde todos los campos incluyendo la academia como lugar de reflexión.

El informe de UNESCO, "La intersección de dos pandemias: la COVID-19 y la violencia contra la mujer”, en julio de 2020 ya advertía varios factores a tener en cuenta y que estaban comenzando a afectar a las mujeres durante la pandemia. Entre las observaciones se destacan elementos que atraviesan las distintas intersecciones de la vida de la mujer y cómo terminan por aumentar la desigualdad de género, ampliando aún más la brecha con el género masculino.

El cierre de las escuelas y las instituciones educativas ha dado lugar a que millones de mujeres se hayan quedado en sus domicilios. El informe señala que, al tener a todos o la mayoría de los miembros del hogar en confinamiento, fueron las mujeres quienes llevaron el mayor peso de la convivencia, debido a que además de realizar sus actividades escolares o de trabajo remoto, eran las encargadas de realizar las tareas de alimentación y limpieza del hogar. Esta carga adicional, ayudó a incrementar la desigualdad frente a sus pares que mayormente se dedicaron a enfocarse en sus estudios o trabajo.

Pedraza Bucio (2021) sostiene en "La brecha digital de género como vértice de las desigualdades de las mujeres en el contexto de la pandemia por Covid-19" que el $48 \%$ de los hogares mexicanos con jefatura femenina y el $47 \%$ de los hogares con jefatura masculina no tienen acceso a Internet; en ambos casos, la principal razón es la falta de recursos económicos, 
Aunque solo hay un punto porcentual de diferencia entre ambos tipos de familia, al considerar que la brecha salarial de género oscila entre el 18.2\% y 22.7\% (INEGI, 2019 b), se entiende que mantener el hogar conectado a Internet representa un gasto mayor para los familias encabezadas por mujeres, que de acuerdo con los resultados de la Encuesta Nacional de Ingresos y Gastos de los Hogares (ENIGH) representa el $28.7 \%$ de las familias del país (INEGI, 2018), Es decir, en una cuarta parte de los hogares mexicanos el gasto en el servicio a Internet es mayor, aunque paguen el mismo precio.

Esta realidad impacta de manera frontal el presente y futuro de la mujer, tanto en el campo escolar como profesional/laboral. El peso de la crisis recae en los hombros de las mujeres, afectando sus aspiraciones, sueños, reproduciendo el ciclo de pobreza, opresión y exclusión. En este sentido, es evidente que la mujer está siendo rezagada a causa de esta falta de oportunidades (Scuro y Bercovich, 2014).

Por este motivo, también se valora las acciones que surgen desde la sociedad civil y gobiernos que trabajan para mejorar las condiciones de exclusión en pro de la igualdad. Además, son importantes las iniciativas como el movimiento STEM (Science, Technology, Engineering and Mathematics), así como las luchas feministas que intentan fomentar vocaciones tecnológicas entre las chicas en edades previas a la universidad (Mosatche et al., 2013; Reinking y Martin, 2018; Mateos y Gómez, 2019).

\subsection{Competencia digital}

El surgimiento de nuevas tecnologías y el desarrollo de software ha permitido crear una amplia gama de técnicas y herramientas que también se adaptan a las necesidades de la educación. Sin embargo, se debe tener en cuenta que las nuevas tecnologías no fueron concebidas para la educación; "no aparecen naturalmente en los sistemas de enseñanza; no se adaptan fácilmente al uso pedagógico y, muy probablemente, en el futuro se desarrollaran solo de manera muy parcial en función de demandas provenientes del sector educacional” (Bonilla, 2003, p. 120).

En este sentido, implantar las TIC en la educación y en las instituciones de Educación Superior, requieren diversos factores a tener en cuenta y que están directamente relacionados con las competencias digitales de docentes y educandos. Bonilla resalta dos elementos o lógicas que han ayudado a implantar estos elementos foráneos en la educación:

En este proceso de 'fuera' hacia 'dentro' del sistema educacional la dimensión temporal es clave: los cambios generados por la incorporación de las TIC a la educación no son inmediatos ni fáciles de identificar. Se trata de un proceso complejo que solo da frutos a medio y a largo plazo. Por otro lado, cabe destacar que dos tipos de lógicas han permitido reducir la exterioridad inicial de las TIC: la lógica de aprender de la tecnología, proporcionando conocimientos acerca de las TIC y sus códigos; y la lógica de aprender con la tecnología, poniendo la tecnología al servicio de los procesos de enseñanzaaprendizaje (Bonilla, 2003, p. 120). 
Para este cometido, es imprescindible un nivel adecuado de conocimientos sobre las TIC, puesto que se asume que "la innovación educativa en educación superior implica creatividad de los docentes, pero requiere apoyo institucional para que se pueda diseminar, escalar e institucionalizar" (Sánchez-Mendiola y Escamilla de los Santos, 2018, p. 38). Sin embargo, todavía, el nivel de conocimiento que permita el fluir de las TIC en la educación limita la potencialidad de los procesos de enseñanza-aprendizaje, puesto que los docentes evitan o restringen el uso de tecnologías; estos factores suelen estar relacionados con la tecnología características del profesorado, por ejemplo, las actitudes y la autoeficacia de los profesores (Herman, Tondeur, van Braak y Valcke; 2008; Peralda et al., 2007; Teo et al., 2008).

Con la declaratoria de Pandemia por parte de la Organización Mundial de la Salud (OMS), en el año 2020 las escuelas, colegios e instituciones de Educación Superior (IES), tuvieron que optar por trasladar las clases hacia la virtualidad y de esta manera continuar con la planificación educativa. Diferentes problemáticas surgieron y afectaron la continuidad y posteriormente la denominada nueva "normalidad", puesto que no se trataba simplemente de utilizar medios para comunicarse como Google Meet, Zoom, Microsoft Teams, etc., sino que se necesitó una nueva estrategia, una nueva forma de entender la educación. Dorencele-Acosta sugiere que:

La innovación educativa en y desde las TIC, exige una infraestructura organizacional (en sus dimensiones de expresión individual, grupal, y social, sintetizado en lo institucional) que va desde la relación docente-estudiante, el capital intelectual de los profesores, sus competencias pedagógicas, digitales e innovadoras, hasta la responsabilidad social, capacidad de resiliencia, gestión de la incertidumbre y adaptación al cambio de la institución (2021, p. 146).

Para ejemplificar esta realidad, según Díaz Barriga (2020), en el caso del profesorado mexicano faltan procesos de formación, ya que solo el $2 \%$ del cuerpo colegiado está capacitado para trabajar digitalmente en la educación básica. Hacia el sur del continente, Ferrada-Bustamante (2021), estima que en Chile cerca del 70\% del profesorado ha tenido capacitación para el uso de TIC en el aula, mientras Vilela et al. (2020) muestran que en Perú las capacitaciones institucionales a docentes incluyen recomendaciones de forma (qué plataformas virtuales emplear) más que de fondo (cómo adaptar su contenido a la educación virtual). En Colombia, Tobar (2017) indica que los profesores tienen un manejo satisfactorio de los recursos tecnológicos, aunque en la competencia pedagógica se aprecia un índice bajo, por lo que el autor de la investigación sugiere que los programas de formación docente en competencias TIC, además de la parte instrumental, deben tener un fundamento epistemológico en modelos. Esto evidencia la importancia de la capacitación docente en tiempos de la Covid-19, con énfasis en el conocimiento de las herramientas digitales, el diseño instruccional en las plataformas en línea, las formas de evaluación y la creación de contenidos, entre otros.

Finalmente, Romero Alonso (2021) en referencia a los estudiantes, muestra que para un $52 \%$ de los alumnos ha sido compleja su adaptación al cambio de metodología de aprendizaje a un formato e-learning de emergencia, al identificarse con emociones negativas, indicando que se sienten abrumados (36\%) y ansiosos (16\%). Además, se encontró una correlación entre la competencia en TIC y las actitudes del profesorado. Sin embargo, aunque la mayoría tiene actitud positiva, cerca de $30 \%$ no las considera necesarias. 
Deroncele-Acosta nos recuerda que la introducción de TIC en educación al no ser natural se servirá del feedback de docentes y éstos de capacitación debido a la "manifiesta necesidad de actualización en innovaciones didácticas desde las TIC, herramientas digitales para el diseño, dinámica y evaluación del E-learning, así como incorporar a la práctica pedagógica recursos didácticos interactivos online" (2021, p. 154).

\subsection{Conectividad y pertinencia}

El Observatorio Regional de Banda Ancha (ORBA) de la Comisión Económica para América Latina y el Caribe (CEPAL) con base en las encuestas de hogares del Banco de Datos de Encuestas de Hogares (BADEHOG), demostró que solo el $67 \%$ de los habitantes y el $60 \%$ de los hogares de la región usa internet, $33 \%$ de los hogares urbanos no está conectado, $77 \%$ de los hogares rurales no está conectado, $42 \%$ de los menores de 25 años y $54 \%$ de los mayores de 66 años no tienen conexión, más de 40 millones de hogares no conectados: la mitad se ubican en los dos quintiles más pobres, 2/3 de los países no alcanzan los requerimientos de velocidad de descarga necesarios para desarrollar soluciones digitales, más de 32 millones de niños y niñas no pueden acceder a soluciones de teleeducación.

En el caso ecuatoriano, antes de la pandemia ya se experimentaba un aumento de personas que podían acceder a internet, como se aprecia en la tabla 1, este aumento se explica por las mejoras en la conectividad nacional, la estabilidad económica y creciente clase media del país.

Tabla 1. Porcentaje de la población ecuatoriana que tiene acceso a Internet

\begin{tabular}{|c|c|}
\hline Año & Indicador \\
\hline 2008 & $25.7 \%$ \\
\hline 2009 & $24.6 \%$ \\
\hline 2010 & $29.0 \%$ \\
\hline 2011 & $31.4 \%$ \\
\hline 2012 & $35.1 \%$ \\
\hline 2013 & $40.4 \%$ \\
\hline 2014 & $46.4 \%$ \\
\hline 2015 & $50.5 \%$ \\
\hline 2016 & $55.6 \%$ \\
\hline
\end{tabular}

Fuente: INEC, 2016

Sin embargo, según el mismo INEC, observa que, si bien existe un aumento de nuevos accesos, el uso del internet en cuanto a la educación o aprendizaje. Es así que mientras en 2008 el 40.1\% estaba destinado a este uso, en 2016 solamente el 23.3\% estaba dedicando el uso de internet a este rubro, lo que podría entenderse como el acceso a internet principalmente para otros sectores o campos de la vida. Sin embargo, el surgimiento de la pandemia aceleró el uso en todo la región, así entre el primer y segundo trimestre de 2020, el uso de soluciones de teletrabajo aumentó 324\% y la educación a distancia más de 60\%, avances que se preveía que demorarían años, pero que se han producido en pocos meses.

Para evitar la desigualdad digital, no solo es importante disponer de equipamiento tecnológico y conectividad sino el tipo de ordenadores que poseen los estudiantes -uso propio 
o compartido con otros familiares o con otros estudiantes-y el tipo de conectividad (Fernández Enguita, 2016). En este sentido es imprescindible aumentar la conectividad, infraestructura y habilidades digitales de maestros y profesores, cerrar la brecha en cuanto al acceso a internet. Los Estados deben adoptar medidas como las que plantea la CEPAL, que propone invertir $1 \%$ del PIB en una canasta básica digital para garantizar el acceso a internet. Estas medidas deben estar acompañadas de soluciones que ayuden a los sectores que tienen acceso a internet pero que es limitado o de baja velocidad, además de entregar dispositivos electrónicos a quienes realmente les es imposible acceder a estas herramientas.

\section{Conclusión}

La segunda década del siglo XXI ha expuesto al mundo a un acelerado cambio a todo nivel, la pandemia obligó a las instituciones educativas a implementar programas urgentes para mantener la continuidad de la programación educativa ahora en un formato enteramente virtual. De lo trabajado en este artículo, se ha podido recoger datos que permiten conocer la realidad de estos cambios y provocan el interés en profundizar investigaciones puntuales sobre las experiencias y problemáticas de las TIC en la educación.

Con preocupación se ha podido constatar que la brecha digital de género es todavía un factor importante y que sigue alimentando la desigualdad a nivel educativo, pero también que esto se extiende a otros campos como el laboral, ya que al dejar por fuera a la mujer o limitarla en el acceso, uso y competencias de las tecnologías, las convertirá fácilmente en el sector que más pierda sus trabajos o no encuentren puestos de trabajo, además; se ha podido constatar que todavía existe un bajo nivel de competencia digital entre los docentes y que repercute en los recursos que se emplean para impartir clases de modo virtual, puesto que además de conocimiento en tecnologías se requiere una actitud positiva o clima de equilibrio para que este nuevo uso de TIC no resulte abrumador en los estudiantes. Finalmente se ha destacado que todavía el acceso a internet para fines educativos es limitado y que necesita ser abordada como política de estado para que la brecha de acceso pueda ser reducida.

\section{Referencias}

Arredondo Trapero, F., Vázquez Parra, J., \& Velázquez Sánchez, L. (2019). STEM y Brecha de Género en Latinoamérica. Revista De El Colegio De San Luis, 9(18), 137-158. https://doi.org/10.21696/ rcsl9182019947

Bonilla, J. (2003), Políticas nacionales de educación y nuevas tecnologías: el caso de Uruguay. En J. Brunner y J. Tedesco. Las nuevas tecnologías y el futuro de la Educación. (pp. 117-127). IIPE-UNESCO.

CEPAL (2020). Universalizar el acceso a las tecnologías digitales para enfrentar los impactos del COVID-19. CEPAL, ONU

Cuban, L. (1986) Teachers and Machines: The Classroom Use of Technology Since 1920. Teachers College Press.

Cuban, L. (2001) Oversold and underused: computers in the classroom. Harvard University Press.

Cuban, L. (2002) Oversold and Underused: Computers in the Classroom. Harvard University Press.

Deroncele-Acosta, Ángel, Medina-Zuta, P., Goñi-Cruz, F. F., Montes-Castillo, M. M., Roman-Cao, E., \& Gallegos Santiago, E. (2021). Innovación Educativa con TIC en Universidades Latinoamericanas: Estudio Multi-País. REICE. Revista Iberoamericana Sobre Calidad, Eficacia Y Cambio En Educación, 19(4), 
145-161. https://doi.org/10.15366/reice2021.19.4.009

Dexter, S. L., Anderson, R. E. \& Becker, H. J. (1999) Teachers' views of computers as catalysts for changes in their teaching practice. Journal of Research on Computing in Education, 31(3), 221-239.

Díez-Gutiérrez, E. J. (2021). Gobernanza híbrida digital y Capitalismo EdTech: la crisis del COVID-19 como amenaza. Foro de Educación, 19(1), 105-133. http://dx.doi.org/10.14516/fde.86o

Dominguez Alonso, J., \& Portela Pino, I. (2020). Violencia a través de las TIC: comportamientos diferenciados por género. RIED. Revista Iberoamericana De Educación a Distancia, 23(2), 273-286. https://doi.org/10.5944/ried.23.2.25916

Engel, A., y Coll, C. (2022). Entornos híbridos de enseñanza y aprendizaje para promover la personalización del aprendizaje. RIED. Revista Iberoamericana de Educación a Distancia, 25(1), 225-242. https://doi. org/10.5944/ried.25.1.31489

Ferrada-Bustamante, V., González-Oro, N., Ibarra-Caroca, M., Ried-Donaire, A., Vergara-Correa, D., \& Castillo-Retamal, F. (2021). Formación docente en TIC y su evidencia en tiempos de COVID-19. Revista Saberes Educativos, (6), 144-168. http://doi.org/10.5354/2452-5014.2021.60715

García Vélez, K. A., Ortiz Cárdenas, T., \& Chávez Loor, M. D. (2021). Relevancia y dominio de las competencias digitales del docente en la educación superior. Revista Cubana de Educación Superior, 40(3), e2o. http://scielo.sld.cu/scielo.php?script=sci_arttext\&pid=S0257-43142021000300020\&lng=es\&tlng=es

González Fernández, M. O. (2021). La capacitación docente para una educación remota de emergencia por la pandemia de la COVID-19. Tecnología, Ciencia y Educación, 19, 81-102. https://doi.org/10.51302/ tce. 2021.614

Gudmundsdottir, G. B. (2010). When does ICT support education in South Africa? The importance of teachers' capabilities and the relevance of language. Information Technology for Development, 16(3), 174-190. doi:10.1080/02681102.2010.498409

Gudmundsdottir, G. B. (2010). When does ICT support education in South Africa? The importance of teachers' capabilities and the relevance of language. Information Technology for Development, 16(3), 174-190. http://doi.org/10.1080/02681102.2010.498409

Jiménez-Becerra, I., \& Segovia-Cifuentes, Y. (2020) Models of didactic integration with ICT mediation: some innovation challenges in teaching practices. Culture and Education, 32(3), 399-440, http://doi. org/10.1080/11356405.2020.1785140

Jimoyiannis, A., \& Komis, V. (2007). Examining teachers' beliefs about ICT in education: implications of a teacher preparation programme. Teacher Development, 11(2), 149-173. http://doi. org/10.1080/13664530701414779

Jonassen, D. H. (2000) Computers as mind tools for schools. Prentice Hall.

INEC. (2016). Tecnologías de la Información y Comunicaciones (TIC'S) 2016 Encuesta Nacional de Empleo Desempleo y Subempleo. INEC.

Lang, M. (2000) Teacher development of computer use in education in Germany. Education and Information Technology, 5(1), 39-48.

Mateos, S., \& Gómez, C. (2019). Libro Blanco de las mujeres en el ámbito tecnológico. Secretaría de Estado para el Avance Digital. Ministerio de Economía y Empresa.

Mosatche, H., Matloff-Nieves, S., Kekelis, L., \& Lawner, E. (2013). Effective STEM programs foradolescent girls: three approaches and many lessons learned. Afterschool matters, 17, 17-25.

Pavez, I. (2015). Niñas y mujeres de América Latina en el mapa tecnológico: Una mirada de género en el marco de políticas públicas de inclusión digital. Cuaderno SITEAL. (diciembre) 1-36 
Pedraza Bucio, C. I. (2021). La brecha digital de género como vértice de las desigualdades de las mujeres en el contexto de la pandemia por Covid-19. LOGOS Revista de Filosofía, 136(49), 9-22. https://doi. org/10.26457//rf.v136i136.2873

Pelgrum, W. J. (2001) Obstacles to the integration of ICT in education: results from a worldwide educational assessment. Computers \& Education, 37, 163-178.

Player-Koro, C. (2012). Factors Influencing Teachers' Use of ICT in Education. Education Inquiry, 3(1), 93-108. doi:10.3402/edui.v3i1.22015

Reinking, A., \& Martin, B. (2018). The Gender Gap in STEM Fields: Theories, Movements, and Ideas to Engage Girls in STEM. Journal of New Approaches in Educational Research, 7(2), 148-153. https://doi. org/10.7821/naer.2018.7.271

Romero Alonso, R., Tejada Navarro, C., \& Núñez, O. (2021). Actitudes hacia las TIC y adaptación al aprendizaje virtual en contexto COVID-19, alumnos en Chile que ingresan a la educación superior. Perspectiva Educacional, 60(2), 99-120. https://dx.doi.org/10.4151/07189729-vol.6o-iss.2-art.1175

Russell, M., Bebell, D., O’Dwyer, L. \& O'Connor, K. (2003) Examining teacher technology use. Implications for preservice and inservice teacher preparation. Journal of Teacher Education, 54(4), 297-310.

Scuro, L., y Bercovich, N. (eds.) (2014). El nuevo paradigma productivo y tecnológico. La necesidad de políticas para la autonomía económica de las mujeres. CEPAL.

Selwyn, N. \& Fitz, J. (2001) The politics of connectivity: The role of big business in UK education technology policy. Policy Studies Journal 29(4), 551-570.

UNESCO (2020, julio 24). La intersección de dos pandemias: la COVID-19 y la violencia contra la mujer. UNESCO. https://cutt.ly/YOaqWld

Vergés Bosch, N., Freude, L., Camps Calvet, C., \& Collado Sevilla, A. (2021). Aprendizaje Servicio, Género y TIC. De la desigualdad de género en las TIC a la generación de vocaciones tecnológicas en el ámbito educativo. Foro de Educación, 19(2), 221-243. https://doi.org/10.14516/fde.885

Vilela, P., Sánchez, J., \& Chau, C. (2021). Desafíos de la educación superior en el Perú durante la pandemia por la covid-19. Desde el Sur, 13(2), e0016. https://dx.doi.org/10.21142/des-1302-2021-0016

Waite, S. (2004) Tools for the job: a report of two surveys of information and communications technology training and use for literacy in primary schools in the West of England. Journal of Computer Assisted Learning, 20, 11-20.

Webb, M. E. (2005) Affordances of ICT in science learning: implications for an integrated pedagogy. International Journal of Science Education, 27(6), 705-735.

World Economic Forum (WEF) (2016). The Global Gender Gap Report. World Economic Forum. http:// reports.weforum.org/global-gender-gap-report-2016

\section{AUTORAS}

Bélgica Cecilia Arias Macías. Magíster en Contabilidad y Finanzas, de la Universidad de Especialidades Espíritu Santo. Contadora publica autorizada de la Universidad Técnica Estatal de Quevedo.

Carmen Luisa Soto Montoya. Magíster en Administración Pública, de la Universidad de Especialidades Espíritu Santo. Magíster en Educación Superior, de la Universidad de Guayaquil. Licenciada en Ciencias de la Educación de la Universidad Técnica de Babahoyo.

Emma Elizabeth Sacon Martinez. Magíster en Dirección del Talento Humano, de la Universidad de Especialidades Espíritu Santo. Ing. En Gestión Empresarial de la Universidad Técnica Estatal de Quevedo. Docente de Educación Superior. 